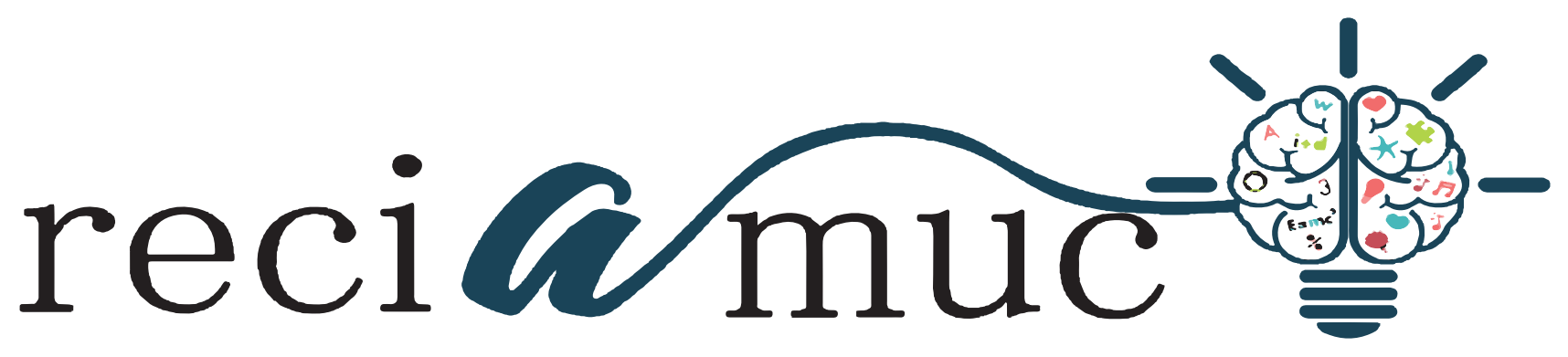

DOI: 10.26820/reciamuc/5.(4).noviembre.2021.163-171

URL: https://reciamuc.com/index.php/RECIAMUC/article/view/739

EDITORIAL: Saberes del Conocimiento

REVISTA: RECIAMUC

ISSN: 2588-0748

TIPO DE INVESTIGACIÓN: Artículo de Revisión

CÓDIGO UNESCO: 32 Ciencias Médicas

PAGINAS: $163-171$

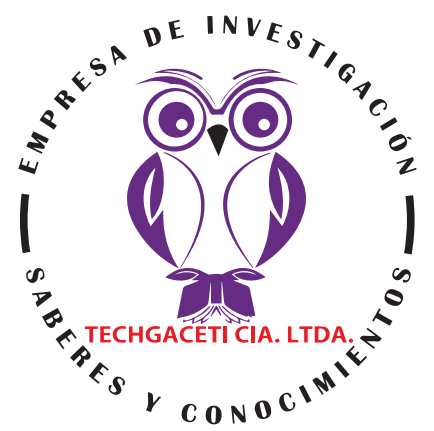

\title{
Efecto del Bypass gástrico en pacientes con Diabetes Mellitus Tipo I
}

\author{
Effect of gastric bypass in patients with Type I Diabetes Mellitus \\ Efeito do bypass gástrico em pacientes com Diabetes Mellitus Tipo I \\ Juan Sebastián de Mora Flores'; Jessenia Estefanía Toalombo Lombeida2; \\ Elizabeth del Rocío Gavilánez Toalombo ${ }^{3}$; María Angiela Goyes Faieta ${ }^{4}$
}

\section{RECIBIDO: 15/09/2021 ACEPTADO: 05/10/2021 PUBLICADO: 29/11/2021}

1. Médico de la Universidad de Guayaquil; Investigador Independiente; Guayaquil, Ecuador; sebastian_demora@hotmail.com; iD https://orcid.org/0000-0001-5493-3955

2. Médico General de la Escuela Superior Politécnica de Chimborazo; Investigador Independiente; Guaranda, Ecuador; yessystef@hotmail.com; iD https://orcid.org/0000-0001-7926-9296

3. Médico General de la Escuela Superior Politécnica de Chimborazo; Investigador Independiente; Guaranda, Ecuador; eliznega_12@hotmail.es; iD https://orcid.org/0000-0003-0437-327X

4. Médica Cirujana de la Pontificia Universidad Católica del Ecuador; Investigador Independiente; Guaranda, Ecuador; angiela.goyes@gmail.com; iD https://orcid.org/0000-0002-0593-5716

\section{CORRESPONDENCIA}

Juan Sebastián de Mora Flores

sebastian_demora@hotmail.com

\section{Guayaquil, Ecuador}

(C) RECIAMUC; Editorial Saberes del Conocimiento, 2021 


\section{RESUMEN}

La obesidad es uno de los principales problemas de salud pública a nivel global. Las alarmantes tasas de sobrepeso y obesidad, y su tendencia al aumento deben impulsar la búsqueda de soluciones. Más de cuatro millones de personas mueren cada año por causas relacionadas con el sobrepeso o la obesidad. El sobrepeso puede dar lugar a la diabetes y sus trastornos conexos, en particular la ceguera, las amputaciones de extremidades y la necesidad de diálisis. En la búsqueda de nuevas modalidades terapéuticas de la diabetes, la cirugía bariátrica ha constituido la manera quirúrgica por medio de la cual se ha logrado la resolución o remisión de la diabetes. Con los años la cirugía bariátrica ha sufrido diversas modificaciones, de las cuales surgió el Bypass Gástrico, cuyo uso ha ido en aumento respecto a otras cirugías bariátricas, siendo en la actualidad una de las más realizadas en todo el mundo. En consecuencia, el propósito fundamental del presente estudio consiste en plasmar los efectos del Bypass gástrico en pacientes con diabetes mellitus tipo 1 (DM1). El modelo de investigación es una revisión de tipo documental bibliográfico. De la revisión se desprende que el Bypass gástrico en pacientes con DM1, mejora diferentes parámetros de la enfermedad, tales como los requerimientos de insulina y la hemoglobina glicada, de lo que se deduce que la remisión o curación de la diabetes se produce por diversos mecanismos, no necesariamente implicados con la pérdida de peso. Asimismo, es recomendable tras esta cirugía bariátrica que los pacientes mantengan un control glucémico óptimo para prevenir complicaciones a largo plazo relacionados con la enfermedad.

Palabras clave: Efecto, Impacto, Bypass gástrico, Diabetes Mellitus, Tipo 1.

\section{ABSTRACT}

Obesity is one of the main public health problems globally. The alarming rates of overweight and obesity and their increasing trend should drive the search for solutions. More than four million people die each year from causes related to being overweight or obesity. Being overweight can lead to diabetes and its related disorders, particularly blindness, limb amputations, and the need for dialysis. In the search for new therapeutic modalities for diabetes, bariatric surgery has been the surgical method by which the resolution or remission of diabetes has been achieved. Over the years, bariatric surgery has undergone various modifications, from which the Gastric Bypass arose, whose use has been increasing compared to other bariatric surgeries, being currently one of the most performed in the world. Consequently, the main purpose of this study is to capture the effects of gastric bypass in patients with type 1 diabetes mellitus (DM1). The research model is a bibliographic documentary type review. The review shows that gastric bypass in patients with DM1 improves different parameters of the disease, such as insulin requirements and glycated hemoglobin, from which it is deduced that the remission or cure of diabetes occurs by various mechanisms, not necessarily involved with weight loss. Likewise, it is recommended after this bariatric surgery that patients maintain optimal glycemic control to prevent long-term complications related to the disease.

Keywords: Effect, Impact, Gastric Bypass, Diabetes Mellitus, Type 1.

\section{RESUMO}

A obesidade é um dos principais problemas de saúde pública mundial. Os alarmantes índices de sobrepeso e obesidade e sua tendência de aumento devem impulsionar a busca por soluções. Mais de quatro milhões de pessoas morrem a cada ano de causas relacionadas ao sobrepeso ou obesidade. O excesso de peso pode causar diabetes e doenças relacionadas, especialmente cegueira, amputações de membros e necessidade de diálise. Na busca por novas modalidades terapêuticas para o diabetes, a cirurgia bariátrica tem sido o método cirúrgico pelo qual se consegue a resolução ou remissão do diabetes. Ao longo dos anos, a cirurgia bariátrica passou por várias modificações, das quais surgiu o Bypass Gástrico, cuja utilização vem crescendo em comparação com outras cirurgias bariátricas, sendo atualmente uma das mais realizadas no mundo. Consequentemente, o objetivo principal deste estudo é capturar os efeitos do bypass gástrico em pacientes com diabetes mellitus tipo 1 (DM1). O modelo de pesquisa é uma revisão bibliográfica do tipo documental. A revisão mostra que o bypass gástrico em pacientes com DM1 melhora diversos parâmetros da doença, como as necessidades de insulina e hemoglobina glicada, da qual se deduz que a remissão ou cura do diabetes ocorre por diversos mecanismos, não necessariamente envolvidos com a perda de peso. Da mesma forma, é recomendado após esta cirurgia bariátrica que os pacientes mantenham um controle glicêmico ideal para prevenir complicações de longo prazo relacionadas à doença.

Palavras-chave: Efeito, impacto, desvio gástrico, diabetes mellitus, tipo 1. 


\section{Introducción}

La obesidad se ha convertido en una verdadera pandemia del siglo XXI, representando uno de los principales problemas de salud pública en todo el mundo. La obesidad y el sobrepeso se definen como una acumulación anormal o excesiva de grasa que puede ser perjudicial para la salud. Un índice de masa corporal (IMC) superior a 25 se considera sobrepeso, y superior a 30, obesidad. Más de cuatro millones de personas mueren cada año por causas relacionadas con el sobrepeso o la obesidad, según estimaciones de 2017 sobre la carga mundial de morbilidad. Asimismo, el sobrepeso puede dar lugar a la diabetes y sus trastornos conexos, en particular la ceguera, las amputaciones de extremidades y la necesidad de diálisis. Desde 1980 las tasas de incidencia de diabetes se han cuadruplicado en todo el mundo. (Organización Mundial de la Salud - OMS, 2021)

Desde 1975, la obesidad se ha casi triplicado en todo el mundo. En 2016, más de 1900 millones de adultos de 18 o más años tenían sobrepeso, de los cuales, más de 650 millones eran obesos. En 2016, el 39\% de las personas adultas de 18 o más años tenían sobrepeso, y el $13 \%$ eran obesas. La mayoría de la población mundial vive en países donde el sobrepeso y la obesidad se cobran más vidas de personas que la insuficiencia ponderal. En 2016, 41 millones de niños menores de cinco años tenían sobrepeso o eran obesos. En 2016 había más de 340 millones de niños y adolescentes (de 5 a 19 años) con sobrepeso $u$ obesidad. (OMS, 2021)

La diabetes mellitus (DM) es una enfermedad que se caracteriza por la alteración del metabolismo glucémico y de los lípidos. Existen varios tipos de DM. La DM1 abarca $5-10 \%$ de todos los tipos de diabetes y la DM2 que se presenta en $90-95 \%$ de todos los tipos de diabetes. La DM1 resulta de la destrucción celular autoinmune de células $\beta$ pancreáticas que generalmente progresa a deficiencia absoluta de insulina. Hay diabetes idiopáticas que son formas de DM1 que no se conocen sus etiologías. Los pacientes tienen insulinopenia y son propensos a cetoacidosis sin que tengan antecedentes de autoinmunidad. (Reyes Ortiz, 2015, p. 2 , 180)

La diabetes tipo 1, antes conocida como diabetes juvenil o diabetes insulinodependiente, es una afección crónica en la que el páncreas produce poca o ninguna insulina por sí mismo. Aproximadamente 62 millones de personas en las Américas (422 millones de personas en todo el mundo) tienen diabetes, la mayoría vive en países de ingresos bajos y medianos, y 244.084 muertes (1.5 millones en todo el mundo) se atribuyen directamente a la diabetes cada año. Tanto el número de casos como la prevalencia de diabetes han aumentado constantemente durante las últimas décadas. (Organización Panamericana de la Salud - OPS, 2021)

La cirugía bariátrica (CB) o cirugía de pérdida de peso implica hacer cambios en el sistema digestivo para ayudar al paciente a perder peso. La cirugía bariátrica se realiza cuando la dieta y el ejercicio no han funcionado o cuando existen problemas graves de salud con ocasión del peso. Algunos procedimientos limitan la cantidad que el paciente puede comer. Otros procedimientos funcionan reduciendo la capacidad del cuerpo para absorber nutrientes. Algunos procedimientos hacen ambas cosas. Aunque la cirugía bariátrica puede ofrecer muchos beneficios, todas las formas de cirugía de pérdida de peso son procedimientos importantes que pueden presentar riesgos y efectos secundarios graves. Entre los tipos de cirugía bariátrica se encuentran: la derivación biliopancreática con cruce duodenal, la gastrectomía en manga y el bypass gástrico (en Y de Roux). (Clínica Mayo, 2021)

El baipás gástrico, también denominado "baipás gástrico en Y de Roux", es un tipo de cirugía para bajar de peso que consiste 
en crear una pequeña bolsa desde el estómago y conectar la bolsa recién creada directamente con el intestino delgado. Después del baipás gástrico, los alimentos ingeridos entrarán en esta pequeña bolsa del estómago y, a continuación, directamente en el intestino delgado, sin pasar por la mayor parte del estómago y la primera sección del intestino delgado. El baipás gástrico es uno de los tipos de cirugía bariátrica que se realizan con más frecuencia. (Clínica Mayo, 2020)

Como una nueva modalidad en la terapéutica contra la diabetes, la cirugía bariátrica ha constituido la manera quirúrgica por medio de la cual se ha logrado la resolución o remisión de la diabetes. Con los años la cirugía bariátrica ha sufrido diversas modificaciones, de las cuales surgió el Bypass Gástrico, cuyo uso ha ido en aumento respecto a otras cirugías bariátricas, siendo en la actualidad una de las más realizadas en todo el mundo. El bypass gástrico ofrece un excelente balance entre pérdida de peso, riesgo quirúrgico y calidad de vida post-intervención (sobre todo BAGUA al Bypass Gástrico Roux-En-Y). Además, la posibilidad de poder realizar BAGUA a medida, o sea usarla tanto en pacientes diabéticos obesos y no obesos ha mejorado su aceptación, al tiempo que ha permitido disminuir la morbi-mortalidad, la estancia y los costes. En los pacientes con DM1, alcanzaron una notable mejoría presentando desde disminución importante de la medicación hasta en algunos casos la suspensión del mismo, con el simple hecho de hacer un bypass gástrico, por lo que se podría considerar su tratamiento quirúrgico seriamente en pacientes con DM1, debido a la agresividad de la enfermedad por la aparición de complicaciones a edad temprana. (Reyes Ortiz, 2015. p. 22, 23)

En consecuencia, el propósito fundamental del presente estudio consiste en plasmar los efectos del Bypass gástrico en pacientes con diabetes mellitus tipo 1.

\section{Materiales y métodos}

Con la finalidad de desarrollar la presente revisión se procedió a la localización de material bibliográfico digitalizado, que representó la plataforma sobre la cual se estructuró la investigación. Para la localización del mismo fueron necesarios ordenadores con conexión a internet. Esta característica general permite la clasificación del presente estudio como una revisión de tipo documental o bibliográfico.

Dicho material fue ubicado por medio de buscadores tales como Google y Google Académico. Igualmente, se usaron portales web relacionados con el área de la salud y con reconocimiento científico internacional, entre los que tienen el de la Organización Mundial de la Salud - OMS, Organización Panamericana de la Salud - OPS, Clínica Mayo, MedlinePlus y Sociedad Andaluza de Medicina Familiar y Comunitaria - SAMFyC.

El material seleccionado consistió en informes, protocolos, tesis de grado y otras clases de contenidos. Fueron excluidos de la selección aquellos contenidos repetidos, cartas editoriales, anotaciones académicas y todo documento carente de sustento científico o con mínimo nivel de evidencia.

En cuanto al tiempo de investigación, la misma se llevó a cabo durante el mes de octubre de 2021. Para los descriptores de búsqueda se usaron los siguientes: "cirugía bariátrica en diabetes", "bypass gástrico en pacientes diabéticos"; "bypass gástrico en diabetes mellitus tipo 1", "impacto del bypass gástrico en diabetes mellitus tipo 1".

Asimismo, los resultados se filtraron según los criterios de idioma español e inglés, Igualmente, la selección incluyó los trabajos de mayor relevancia y que guardaban una debida correlación temática. El rango de fecha de publicación del material estuvo entre los años 2010 al 2021.

Los autores manifiestan no tener ningún conflicto de intereses. 


\section{Resultados}

La DM es una enfermedad que debe ser tratada de forma multidisciplinaria. Esto debe incluir modificación de la dieta, incremento de actividad física, modificación de la conducta o tratamiento a base de drogas. Sin embargo, a pesar de los esfuerzos por controlar la enfermedad por medio de tratamiento médico, las complicaciones micro- y macro-vasculares no se detienen. Específicamente, el tratamiento de la DM1, se encuentra basado en la combinación de insulina, dieta y ejercicio. No obstante, se ha demostrado que el tratamiento médico no logra detener el avance de la enfermedad. Actualmente existe otra modalidad de tratamiento optando por cirugía gastrointestinal tipo metabólica o de diabetes como tratamiento actual. La resolución de la diabetes se ha demostrado en la literatura mundial a través de múltiples estudios, desde grandes meta-análisis sobre cirugía bariátrica, revisiones y comparaciones de tratamiento médico vs tratamiento quirúrgico. Y recientemente se ha considerado a la cirugía bariátrica como cirugía metabólica y ahora como cirugía de diabetes. (Reyes Ortiz, 2015, p. 183)

El bypass gástrico, que también recibe el nombre de "bypass gástrico en Y de Roux", es un procedimiento quirúrgico en el cual el cirujano crea un pequeño saco en la parte superior del estómago. Este saco se transforma en el nuevo estómago. Posteriormente, este saco se conecta a la parte media del intestino delgado, saltándose la parte superior del intestino delgado. Después de la operación, el saco estomacal puede contener mucha menos comida que un estómago de tamaño normal. El resultado es que el paciente comerá menos cantidad de alimentos, se sentirá más satisfecho que antes y tendrá menos hambre. Dado que el intestino delgado será más corto, absorberá menos calorías y menos nutrientes. Las personas con bypass gástrico suelen perder más peso que las que tienen una manga gástrica, pero también pueden te- ner más problemas. El procedimiento de bypass gástrico es irreversible (ver Figura 1). (Reichard, 2019)

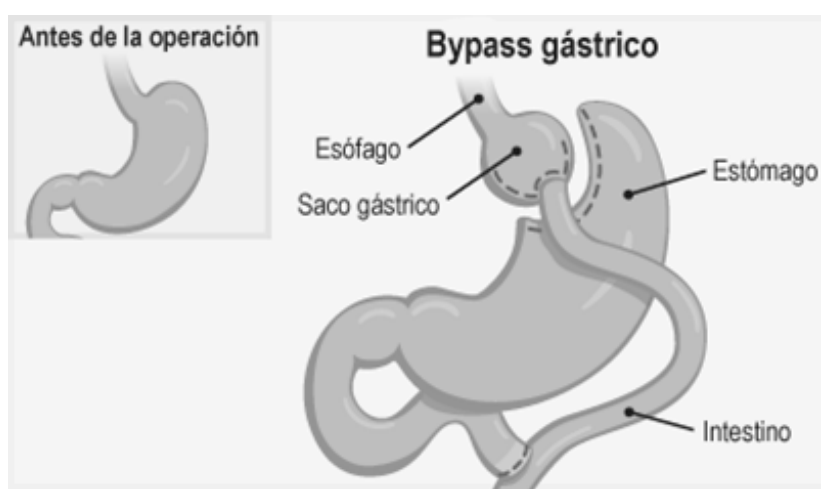

Imagen 1. Bypass gástrico.

Fuente: "Cirugía para perder peso". Reichard, K. (2019). KidsHealth. URL: https:// kidshealth.org/es/teens/bariatric.html

Acerca de la cirugía bariátrica o metabólica, en líneas generales, es importante mencionar entre sus ventajas que permite conseguir la remisión de la diabetes en dos de cada tres pacientes, en especial con la técnica del bypass gástrico en $Y$ de Roux, incluso antes de que se produzca una pérdida de peso significativa. Los mecanismos no están claros, dado que parecieran intervenir varios: aumento de péptidos intestinales similar al glucagón tipo 1 (GLP 1), la reducción brusca del consumo de nutrientes y la mejora de la esteatosis lo que comporta una mejora de la sensibilidad a la insulina y un aumento de su secreción. Este tipo de cirugía se encuentra indicada en personas con un índice de masa corporal (IMC) $\geq 40$ $\mathrm{kg} / \mathrm{m} 2$ o bien con un IMC entre 35 y 39.9 $\mathrm{kg} / \mathrm{m} 2$ con presencia de graves comorbilidades y que se comprometan a realizar un régimen dietético y de ejercicio adecuado. Entre sus principales contraindicaciones se encuentran las enfermedades cardiorrespiratorias significativas que impliquen un alto riesgo anestésico, el incumplimiento terapéutico y los trastornos psicológicos o psiquiátricos importantes. Con relación a sus riesgos y complicaciones se tienen 
los episodios de hipoglucemia por aumento de la secreción de insulina derivada de una respuesta exagerada de la secreción de GLP-1. Por último, en cuanto a las consecuencias nutricionales, la más común es la deficiencia de hierro, seguido del déficit de vitamina B12, la posible deficiencia de tiamina, la cual puede dar lugar a secuelas neurológicas, la de cobre que produce ataxia, neuropatía periférica, cambios en el metabolismo del calcio y déficit de vitaminas liposolubles A, D, E y K. (Grupo Diábetes de la Sociedad Andaluza de Medicina Familiar y Comunitaria - SAMFyC, 2012)

García Caballero, (2010) referido por Reyes Ortiz, (2015), destaca que hoy en día existe un gran debate entre endocrinólogos, científicos básicos y cirujanos, acerca de que la diabetes se puede curar con cirugía bariátrica tipo bypass gástrico, por lo que con los hechos demostrados, los cirujanos no estaban preparados para considerar a la diabetes como una enfermedad curable tras cirugía, y en particular tras bypass gástrico alcanzando una tasa de remisión de hasta el 83\%. Asimismo, manifiestan lo siguiente: "Tras la cirugía, se logra efecto inmediato de mejoría o resolución de DM1 y DM2, dándose de alta del hospital con tratamiento reducido o sin tratamiento antidiabético". (p. 186, 184)

Por su parte, Mateo et al., (2019) manifiestan que existen algunas series de casos publicados sobre el impacto de la cirugía bariátrica en pacientes con obesidad mórbida y diabetes mellitus tipo 1, con aparentes efectos metabólicos beneficiosos, aunque el papel de la cirugía bariátrica en este grupo de pacientes precisa de estudios más a largo plazo. Con respecto a los mecanismos fisiopatológicos implicados, al margen de los incuestionables beneficios que implica la reducción energética y la pérdida ponderal sobre el metabolismo glucémico se han postulado en los últimos años diversas teorías que tratan de explicar sus efectos beneficiosos sobre la resistencia a la insulina y el control glucémico:
Reducción en la absorción de grasas en las técnicas con componente mal absortivo. Lo cual implica una menor oxidación de ácidos grasos libres, disminuye la convergencia de grasas en tejidos sensibles a la insulina como el músculo o el hígado, lo que parece favorecer una mayor sensibilidad a la insulina, así como una menor lipotoxicidad para las células beta pancreáticas. (p. 512)

Existen algunas teorías intestinales que tratan de explicar cómo la cirugía bariátrica produce efectos sobre la diabetes mellitus debido a los cambios sobre algunas hormonas. Reyes Ortiz, (2015) explica al respecto:

Resultados de la cirugía bariátrica en la pérdida de peso, a menudo resulta en la remisión o curación de la diabetes. Sin embargo, la rápida mejora de la glucosa en la sangre dentro de días y/o semanas después sugiere mecanismos independientes del peso en el control de la diabetes. Hay muchas teorías posibles propuestas para explicar esto. La teoría del intestino posterior (The hindgut or incretin hypothesis) propone que la rápida entrega de nutrientes al intestino delgado distal aumenta la liberación de GLP1 y PYY que mejoran el metabolismo de la glucosa. La hipótesis del intestino medio o de la regulación intestinal/hepática (midgut hypothesis or intestinal/hepatic regulation hypothesis) propone que la derivación de nutrientes al intestino delgado distal después de bypass gástrico Roux-en-Y (BGYR) mejora la gluconeogénesis intestinal, activa el sistema de señalización de glucosa hepato-portal, lo que disminuye la ingesta de alimentos y suprime la producción de glucosa hepática (HGP), que lleva a mejorar la homeostasis de la glucosa. La hipótesis del intestino anterior (the foregut hypothesis) propone que la exclusión de los nutrientes en el intestino delgado proximal puede suprimir la secreción de factores anti-incretina desconocidos, dando lugar al aumento de la liberación de las incretinas que mejoran el control de la glucosa. Por lo tanto, las mejoras metabólicas después del bypass gástrico han Ilevado a denominarla como una 
"cirugía metabólica" y a la DM2 a ser etiquetada como "enfermedad intestinal". Sin embargo, la DM1 también mejora de una manera importante tras bypass gástrico como se demuestra en este estudio, por lo que se debería de considerar también como enfermedad intestinal. Las 3 teorías intestinales mencionadas previamente, juegan un papel importante en los pacientes con DM1 no obesos tras cirugía a pesar de que los pacientes no tienen actividad celular beta, la actividad incretínica pudiera provocar neogénesis de los islotes para que apropiadamente secreten insulina, así como otras hormonas endógenas presentes normalmente. (p. 14)

Hasta la fecha es poco lo que se conoce acerca de la cirugía bariátrica en la DM1. Czupryniak et al, (2004) referidos por Raab, Weine, Frenken, Rett, \& Weiner, (2013) han sido unos de los pioneros en informar sobre la cirugía bariátrica en la diabetes mellitus tipo 1. En su estudio observaron a 2 pacientes mujeres a la edad de 23 años y 28 que se sometieron a bypass gástrico. En ambos casos se observó una reducción del IMC (preoperatoria 38,8 / 46,3 kg / m2 y un año después de la cirugía $26,6 / 30,1 \mathrm{~kg} / \mathrm{m} 2$ ) y una mejora de la sensibilidad a la insulina. El requerimiento diario de insulina podría reducirse de 68/120 UI antes de la cirugía a 45/70 UI un año después cirugía. (p. 33)

Posteriormente, Czupryniak et al., (2010) describieron tres pacientes con diabetes tipo 1 mal controlados, de 19, 23 y 28 años, los cuales se sometieron a una cirugía de bypass gástrico en $Y$ de Roux, donde obtuvieron los siguientes resultados: la reducción máxima de peso corporal observada durante el seguimiento fue de entre $27 \%$ y $31 \%$ del peso corporal inicial, el requerimiento de insulina disminuyó de 0,6-0,95 a 0,3-0,83 UI / kg y la reducción absoluta de $\mathrm{HbA} 1 \mathrm{c}$ fue de 3-4\%. Se observó una mejora significativa en la presión arterial, el perfil de lípidos plasmáticos y la microalbuminuria. Los autores concluyeron que, la cirugía de BGYR en pacientes con diabetes tipo 1 con obesidad mórbida conduce a una pérdida de peso significativa y mantenida y da como resultado una mejora notable en el control de la glucosa en sangre y trastornos concomitantes. La cirugía bariátrica debe recomendarse a los pacientes con diabetes tipo 1 significativamente obesos como un medio para reducir el riesgo de complicaciones vasculares.

Mendez, Tanenberg, \& Pories, (2010) informaron en su estudio de 3 pacientes de sexo femenino con DM1 que tenían cirugía de bypass. EI IMC preoperatorio fue 40,6-53,3 $\mathrm{kg} / \mathrm{m} 2$ y la dosis diaria de insulina osciló entre 52,2-180 UI. Un año después de la cirugía, los autores observaron una mejora notable no solo del peso corporal sino también de la sensibilidad a la insulina. EI IMC fue reducido a 26,7-30,8 kg / m2 y la cantidad diaria de la insulina fue de 25,6 a 48,2 UI. En conclusión, el procedimiento bariátrico RYGB parece no mejorar directamente el control glucémico en DM1. El control glucémico es desafiante en estos pacientes, incluso después de disminuir su exceso de peso corporal y mejorar la sensibilidad a la insulina. Se necesitan estudios de casos y controles aleatorios para demostrar resultados más definitivos. (p. 281-283)

Raab et al., (2013) en los resultados de su estudio mostraron para todos los pacientes una notable reducción de peso, así como una mejora en su control de glucosa en sangre y el requerimiento de insulina en el año de seguimiento después de la cirugía. Estos autores explican lo siguiente:

Antes de la cirugía el IMC de nuestros 6 pacientes osciló entre $37,3-43,0 \mathrm{~kg} / \mathrm{m} 2$ y mejoró a 25,3-29,0 kg / m2 un año después de la cirugía. La HbA1c disminuyó del 6,7\% al $9,8 \%$ antes de la cirugía a un 5,7\% a 8,5\% después de un año de postoperatorio. El requerimiento de insulina (unidades por $\mathrm{kg}$ de peso corporal) se redujo de 0,72-1,13 UI / kg antes de la cirugía a 0,14-0,62 UI / kg después un año. La cantidad total de insulina requerida diaria se redujo de 62-150 UI

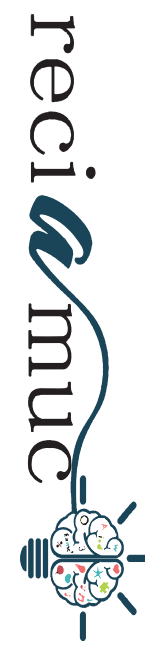


/ día antes de la cirugía a 15-54 UI / día un año después de la cirugía. Solo tenemos pocos datos para la Paciente $\mathrm{C}$ porque interrumpió el seguimiento. En el Paciente A observamos los valores de glucosa en sangre y los requerimientos de insulina durante su estadía en nuestro hospital. La noche antes de la cirugía comenzamos esto control con el CGMS (continuo subcutáneo sistema de control de glucosa). Observamos una mejora de la sensibilidad a la insulina directamente después de la cirugía: el mismo efecto que se describe después de un bypass gástrico cirugía en diabetes mellitus tipo 2 . Los autores llegaron a la siguiente conclusión: la cirugía de obesidad es un método eficaz para controlar el peso, reducción y tratamiento de comorbilidades no solo para diabetes mellitus tipo 2, sino también en pacientes con DM1. No obstante, es recomendable para los pacientes con diabetes tipo 1 tener un control glucémico óptimo para prevenir complicaciones a largo plazo debido a la diabetes. Esto sigue siendo un desafío para todos. (p. 32, 34)

Polo et al., (2019) realizaron una revisión retrospectiva de las historias clínicas de siete pacientes con DM1 y obesidad a quienes se les efectuó una cirugía bariátrica. Los pacientes tenían diagnóstico de obesidad grado II o III previo a la intervención. El diagnóstico de DM1 se confirmó en todos los pacientes por la presencia de pancreatitis autoinmune, ausencia de péptido-C o historia de cetoacidosis diabética. Se evaluó a los pacientes basal y al año de realizada la cirugía. En tres pacientes se realizó bypass gástrico en $Y$ de Roux y gastrectomía en manga en cuatro. Los autores Ilegaron a las siguientes conclusiones:

Los hallazgos de nuestra serie de casos alientan a considerar a la CB como una herramienta terapéutica efectiva en pacientes con DM1 y obesidad, con el objetivo de mejorar el control glucémico y lograr el descenso del peso corporal. Dado que el seguimiento evaluado en estos pacientes fue a un año, se necesitaría un mayor se- guimiento para analizar si la mejoría evidenciada en el control metabólico se sostiene a largo plazo. A la luz de los resultados, sería apropiado considerar a la CB como una opción terapéutica eficaz en pacientes con obesidad y DM1, siendo el principal beneficio el manejo del peso corporal y la consecuencia de la mejoría del peso corporal en los parámetros metabólicos que se derivan de ella. Los resultados a corto plazo de la CB en pacientes con DM1 son alentadores, pero se necesitan estudios con un número más grande de pacientes y mayor seguimiento. (p. 142)

\section{Conclusiones}

De la revisión se desprende que el Bypass gástrico en pacientes con diabetes mellitus tipo 1, mejora diferentes parámetros de la enfermedad, tales como los requerimientos de insulina y la hemoglobina glicada, de lo que se deduce que la remisión o curación de la diabetes se produce por diversos mecanismos, no necesariamente implicados con la pérdida de peso.

El Bypass gástrico, es un método eficaz para controlar el peso y para la reducción y tratamiento de comorbilidades para diabetes mellitus tipo 1. No obstante, estos pacientes deben tener un control glucémico óptimo para prevenir complicaciones a largo plazo relacionados con la enfermedad.

\section{Bibliografía}

Clínica Mayo. (17 de Octubre de 2020). Clínica Mayo. Recuperado el 10 de Octubre de 2021, de https:// www.mayoclinic.org/es-es/tests-procedures/gastric-bypass-surgery/about/pac-20385189\#: :text=Descripci\%C3\%B3n\%20general, directamente\%20con\%20el\%20intestino\%20delgado.

Clínica Mayo. (18 de Septiembre de 2021). Clínica Mayo. Recuperado el 10 de Octubre de 2021, de https://www.mayoclinic.org/es-es/tests-procedures/bariatric-surgery/about/pac-20394258

Czupryniak, L., Wiszniewski, M., Szymański, D., Pawłowski, M., Loba, J., \& Strzelczyk, J. (Abril de 2010). Resultados a largo plazo de la cirugía de bypass gástrico en pacientes con diabetes tipo 1 con obesidad mórbida . Cirugía de obesidad, 20, 
506-508. doi:https://doi.org/10.1007/s11695-0100074-6

Grupo Diábetes de la Sociedad Andaluza de Medicina Familiar y Comunitaria - SAMFyC. (11 de Julio de 2012). Sociedad Andaluza de Medicina Familiar y Comunitaria - SAMFyC. Recuperado el 11 de Octubre de 2021, de http://www.grupodiabetessamfyc.es/index.php/guia-clinica/guia-clinica/ tratamiento/cirugia-y-diabetes.html

Mateo, I., Vílchez, F., Mayo, M., Medina, D., Sánchez, E., Pacheco, J., . . A Aguilar, M. (2019). Resultados metabólicos de la cirugía bariátrica. Impacto sobre el riesgo cardiovascular. Cirugía Andaluza, 30(4), 510-514. Recuperado el 15 de Octubre de 2021, de https://www.asacirujanos.com/admin/upfiles/revista/2019/Cir_Andal_vol30_n4.pdf

Mendez, C. E., Tanenberg, J. R., \& Pories, W. (09 de Agosto de 2010). Resultados de la cirugía de bypass gástrico en $Y$ de Roux para pacientes gravemente obesos con diabetes tipo 1: informe de una serie de casos. Diabetes, síndrome metabólico y obesidad: objetivos y terapia, 3, 281283. Recuperado el 29 de Octubre de 2021, de https://thescholarship.ecu.edu/bitstream/handle/10342/5395/dmso-3-281.PMC3047955.pdf?sequence $=1$ \&isAllowed $=\mathrm{y}$

OMS. (09 de Junio de 2021). Organización Mundial de la Salud - OMS. Recuperado el 05 de Octubre de 2021, de https://www.who.int/es/news-room/ fact-sheets/detail/obesity-and-overweight
Organización Mundial de la Salud - OMS. (2021). Organización Mundial de la Salud - OMS. Recuperado el 08 de Octubre de 2021, de https://www.who. int/es/health-topics/obesity\#tab=tab_1

Organización Panamericana de la Salud - OPS. (2021). Organización Panamericana de la Salud OPS. Recuperado el 20 de Octubre de 2021, de https://www.paho.org/es/temas/diabetes

Polo, P., Álvarez, M., Babor, E., Herrero, M., González, S., Yuma, M., . . . Aguirre, M. (Septiembre-Diciembre de 2019). Impacto de la cirugía bariátrica en pacientes con obesidad y diabetes mellitus tipo 1. Revista de la Sociedad Argentina de Diabetes, 53(3), 139-142. Recuperado el 30 de Octubre de 2021, de https://www.revistasad.com/index.php/ diabetes/article/download/170/149

Raab, H., Weine, R. A., Frenken, M., Rett, K., \& Weiner, S. (2013). Obesidad y cirugía metabólica en diabetes mellitus tipo 1. Nutrición Hospitalaria, 28(2), 31-34. Recuperado el 20 de Octubre de 2021, de https://scielo.isciii.es/pdf/nh/v28s2/06articulo05.pdf

Reichard, K. W. (Noviembre de 2019). KidsHealth. Recuperado el 20 de Octubre de 2021, de https:// kidshealth.org/es/teens/bariatric.html

Reyes Ortiz, A. (2015). Cirugía de Diábetes Tipo 1 y 2. Universidad de Málaga, Facultad de Medicina. Departamento de Especialidades Quirúrgicas y Bioquímica. Málaga: Publicaciones y divulgación científica de la Universidad de Málaga. Recuperado el 15 de Octubre de 2021, de https://riuma. uma.es/xmlui/bitstream/handle/10630/10760/TD_ REYES_ORTIZ.pdf? sequence $=1$ \&isAllowed $=y$

\section{CITAR ESTE ARTICULO:}

de Mora Flores, J. S., Toalombo Lombeida, J. E., Gavilánez Toalombo, E. del R., \& Goyes Faieta, M. A. (2021). Efecto del Bypass gástrico en pacientes con

Diabetes Mellitus Tipo I. RECIAMUC, 5(4), 163-171. https://doi.org/10.26820/ reciamuc/5.(4).noviembre.2021.163-171

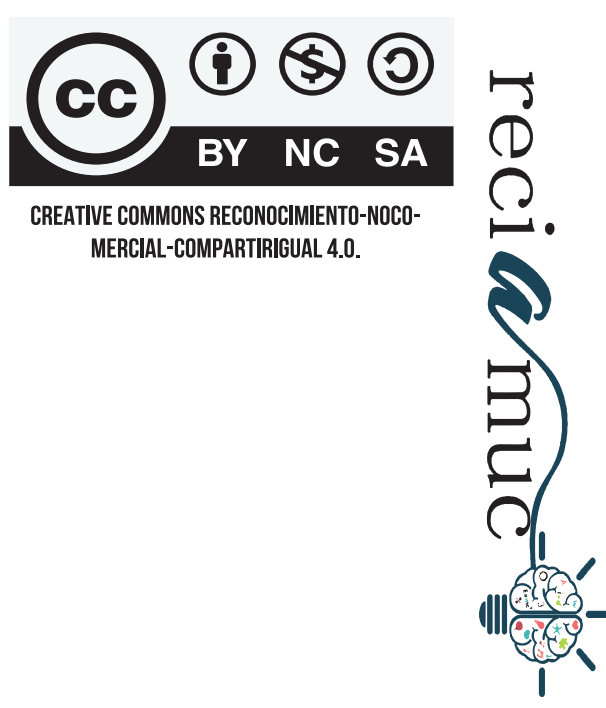

